Pacific

Journal of

Mathematics

ON EMBEDDINGS INTO COMPACTLY GENERATED GROUPS

Pierre-Emmanuel CAPrace AND Yves Cornulier

Volume 269 No. 2

June 2014 


\title{
ON EMBEDDINGS INTO COMPACTLY GENERATED GROUPS
}

\author{
PierRe-EMmanuel CAPRACE AND Yves CoRnulier
}

\begin{abstract}
We prove that there is a second-countable locally compact group that does not embed as a closed subgroup in any compactly generated locally compact group, and discuss various related embedding and nonembedding results.
\end{abstract}

\section{Introduction}

The Higman-Neumann-Neumann (HNN) theorem [Higman et al. 1949] ensures that every countable group embeds as a subgroup of a finitely generated group, indeed 2-generated (relying on a fundamental construction referred to since then as $H N N$-extension). This was a major breakthrough, providing some of the first evidence that finitely generated groups are not structurally simpler than countable groups and thus are far from tame or classifiable. B. H. and H. Neumann [Neumann and Neumann 1959] gave an alternative construction, showing for instance that every countable $k$-solvable group (that is, solvable of derived length at most $k$ ) embeds as a subgroup of a finitely generated $(k+2)$-solvable group. Further refinements by P. Hall [1954] and P. Schupp [1976] in a slightly different direction showed that every countable group embeds in a 2-generated simple group.

In the present paper, we address similar questions in the context of locally compact topological groups, which will be abbreviated henceforth by the term l.c. groups. Recall that locally compact groups are a natural generalization of discrete groups, the counterpart of countability (resp. finite generation), being $\sigma$ compactness (resp. compact generation). A prototypical example of an embedding of a noncompactly generated 1.c. group into a compactly generated one is the embedding of the $p$-adic additive group $\mathbf{Q}_{p}$ into the affine group $\mathbf{Q}_{p} \rtimes \mathbf{Q}_{p}^{\times}$(or its discrete cousin, the embedding of the additive underlying group of the ring $\mathbf{Z}[1 / p]$ into the Baumslag-Solitar group $\mathbf{Z}[1 / p] \rtimes_{p} \mathbf{Z}$ ).

It is natural to ask whether analogues of the HNN theorem hold in the context of 1.c. groups. In that context, an embedding $\varphi: H \rightarrow G$ of an 1.c. group $H$ to an

Caprace was partly supported by FNRS grant F.4520.11 and the European Research Council (grant \#278469). Cornulier was partly supported by ANR grant GSG..

MSC2010: primary 22D05; secondary 05C63, 20E18, 57S30.

Keywords: locally compact groups, compactly generated groups, topologically simple groups, unrestricted wreath products. 
1.c. group $G$ is defined as a continuous injective homomorphism (with potentially nonclosed image). In the nondiscrete setting, several natural variants of the question can be considered:

- Given a $\sigma$-compact 1.c. group $H$, is there any embedding $\varphi: H \rightarrow G$ into a compactly generated 1.c. group $G$ ?

- Is there one with closed image?

- Is there one with open image?

It turns out that whenever the topology on $H$ is nondiscrete, the answers to these questions are not always positive, and depend heavily on the algebraic structure of $H$. The results of this note are intended to illustrate that matter of fact. We start with a positive result in the case where the algebraic structure of $H$ is the simplest possible, namely, when $H$ is abelian:

Theorem 1.1. Every $\sigma$-compact abelian l.c. group A embeds as an open subgroup of a compactly generated group $G$, which can be chosen to be 3-solvable. If moreover $A$ is totally disconnected, second countable, or both, then $G$ can also be chosen to enjoy the same additional property.

In particular, the additive group of adeles, defined as a restricted product of all $\mathbf{Q}_{p}$ (see Section 4 for the definition) is isomorphic to an open subgroup of a compactly generated locally compact group. In contrast, the adeles are used to prove the following result, which shows in particular that Theorem 1.1 cannot be generalized to solvable groups:

Theorem 1.2. There exists a $\sigma$-compact metabelian l.c. group $M$ not isomorphic to any closed subgroup of any compactly generated l.c. group.

Moreover M can be chosen to be second countable and totally disconnected.

The proof of Theorem 1.2 is based on the now classical observation, due to H. Abels [1974, Beispiel 5.2], that every compactly generated 1.c. group admits, in a somewhat natural way, a continuous proper action on a connected graph of bounded degree (see Proposition 2.1 below). Using similar ideas, we obtain the following result, which shows that the HNN theorem fails in the nondiscrete setting, even if one allows embeddings with potentially nonclosed images:

Theorem 1.3. There exists a second-countable (hence $\sigma$-compact) topologically simple totally disconnected l.c. group $S$ such that every continuous (or even abstract) homomorphism of $S$ to any compactly generated l.c. group is trivial.

This stands in sharp contrast with the discrete case. We remark also that local compactness is absolutely essential to this result, since it is known from [Pestov $1986]$ that every $\sigma$-compact topological Hausdorff group is isomorphic to a closed subgroup of some compactly generated topological Hausdorff group. 
We finally present a result illustrating the difference between embeddings with closed and open images:

Theorem 1.4. There exists a second-countable (hence $\sigma$-compact) l.c. group $H$ that is isomorphic to a closed subgroup of a compactly generated l.c. group, but not to any open subgroup of any compactly generated l.c. group.

Moreover, $H$ can be chosen to be of the form $K \rtimes \Gamma$, with $\Gamma$ discrete abelian and $K$ compact abelian and either connected or profinite. It can also be chosen to be a Lie group.

One part of the implication in Theorem 1.4 is the following general fact, which is based on a wreath product construction:

Proposition 1.5. Any compact-by-\{countable discrete\} l.c. group embeds as a closed subgroup in a compact-by-\{finitely generated discrete\} l.c. group.

Similarly to Theorem 1.1, this proposition illustrates that embedding theorems can hold in the nondiscrete case when the algebraic or topological structure of the group $H$ is not too complicated.

We finish by mentioning some related natural questions which we have not been able to answer.

Question 1.6. Is every second-countable (real) Lie group isomorphic to a closed subgroup of a compactly generated locally compact group? Of a compactly generated Lie group? Same questions for $p$-adic Lie groups.

The answer to Question 1.6 with "closed subgroup" replaced by "open subgroup" is negative for both real and $p$-adic Lie groups; see the examples in Section 6.

\section{Locally compact groups, Lie groups, and locally finite graphs}

We shall use the following general result about 1.c. groups; the first part follows from the solution to Hilbert's fifth problem, the second is an elementary but crucial observation due to $\mathrm{H}$. Abels:

Proposition 2.1. Let $G$ be an l.c. group and $V$ be any identity neighborhood.

(i) (Yamabe) If $G$ is connected-by-compact (i.e., if $G / G^{\circ}$ is compact), then $V$ contains a compact normal subgroup $K$ of $G$ such that $G / K$ is a connected Lie group.

(ii) (Abels) If $G$ is totally disconnected and compactly generated, then $V$ contains a compact normal subgroup $W$ of $G$ such that $G / W$ admits a faithful continuous proper vertex-transitive action on some connected locally finite graph. 
Proof. For (i), see [Montgomery and Zippin 1955, Theorem IV.4.6]. For (ii), originally observed in [Abels 1974, Beispiel 5.2], refer to [Monod 2001, §11.3].

We deduce the following useful criterion for the nonexistence of embeddings into compactly generated l.c. groups:

Proposition 2.2. Let $H$ be an l.c. group. The following are equivalent:

(1) Every continuous homomorphism of $H$ to a compactly generated l.c. group is trivial.

(2) The following two conditions are satisfied:

(a) Every continuous homomorphism of $H$ to a compactly generated totally disconnected l.c. group is trivial.

(b) For any $n$, every continuous linear representation $H \rightarrow \mathrm{GL}_{n}(\mathbf{C})$ is trivial. Moreover, a sufficient condition for (a) is that $H$ has no nontrivial continuous action on any connected graph of bounded degree.

Proof. That (1) implies (2) is immediate. Conversely, assume that (2) holds. Let $G$ be a compactly generated 1.c. group and $f: H \rightarrow G$ a continuous homomorphism. Considering the composite map $H \rightarrow G \rightarrow G / G^{\circ}$ in view of (a), we see that $f(H) \subset G^{\circ}$. If $f$ is not the trivial map, some identity neighborhood $V$ in $G$ does not contain the image of $f$. By Proposition 2.1(i), there is a compact normal subgroup $K$ of $G^{\circ}$ contained in $V$ such that $L=G^{\circ} / K$ is a (connected) Lie group. So the composite map $H \rightarrow L$ is nontrivial. Using the adjoint representation of $L$ and (b), we see that it maps $H$ into the center of $L$. On the other hand, it follows from Pontryagin duality and (b) that $H$ admits no nontrivial continuous homomorphism to any abelian 1.c. group. So we get a contradiction, and thus $f$ is the trivial homomorphism.

Let us now assume that $H$ has no nontrivial continuous action on any connected graph of bounded degree and let us check that (a) holds. Let $f: H \rightarrow G$ be a continuous homomorphism, with $G$ a compactly generated totally disconnected 1.c. group. If $f$ is nontrivial, some identity neighborhood $V$ in $G$ does not contain the image of $f$. By Proposition 2.1(ii), there is a compact normal subgroup $K$ of $G$ contained in $V$ such that $G / K$ acts continuously, faithfully, and vertex-transitively on a connected locally finite graph. The hypothesis made on $H$ implies that the restriction of this action to $H$ is trivial. Thus $f(H)$ is contained in $K$, hence in $V$, which is a contradiction.

Remark 2.3. We do not know if, conversely, (a) implies that $H$ has no nontrivial continuous action on any connected graph of bounded degree. In other words, does the existence of a continuous nontrivial action on a connected graph of bounded degree imply the existence of such an action on a vertex-transitive graph? The same question, replacing "nontrivial" by "proper," can also naturally be addressed. 
Finally, we record an elementary fact, allowing us in suitable situations to exclude actions on some connected locally finite graphs:

Lemma 2.4. Let $G$ be an l.c. group acting continuously by automorphisms on a connected graph all of whose vertices have degree $\leq d$. Then every vertex stabilizer $O$ is open in $G$ and, for any prime $p>d$, every closed pro-p subgroup of $O$ acts trivially on the graph. In particular, if $G$ admits an open pro-p-group, then the action has an open kernel.

Proof. Let $O$ be a vertex stabilizer, which is open in $G$ since the action on the graph is assumed continuous. Given any closed subgroup $H$ of $O$ which acts nontrivially on the graph, there is a vertex $v$ fixed by $H$ and adjacent to some vertex that is not fixed by $H$. In particular, $H$ admits some nontrivial continuous permutation action on the set of neighbors of $v$, which is a set of at most $d$ elements. It follows that $H$ cannot be pro- $p$ for any $p>d$.

\section{Proof of Theorem 1.1}

Recall that any totally disconnected 1.c. group contains compact open subgroups. Moreover, every abelian 1.c. group $A$ has a (noncanonical) decomposition as a topological direct product $\mathbf{R}^{n} \times W$, where $W$ is compact-by-discrete, and the discrete quotient is countable as soon as $A$ is $\sigma$-compact. Those facts could be used to deduce (a part of) Theorem 1.1 from Proposition 1.5. This is however not what we shall do here, and we present rather a simpler direct argument.

We begin with an easy classical result:

Lemma 3.1. There exists a finitely generated group $\Gamma$ whose center contains a free abelian group $Z$ of countable rank; $\Gamma$ can be chosen to be 3-solvable.

Proof. If $t$ is an indeterminate, the reader can check that the three matrices

$$
\left(\begin{array}{lll}
1 & 0 & 0 \\
0 & t & 0 \\
0 & 0 & 1
\end{array}\right), \quad\left(\begin{array}{lll}
1 & 1 & 0 \\
0 & 1 & 0 \\
0 & 0 & 1
\end{array}\right), \quad\left(\begin{array}{lll}
1 & 0 & 0 \\
0 & 1 & 1 \\
0 & 0 & 1
\end{array}\right)
$$

generate a group containing the set of all matrices of the form

$$
\left(\begin{array}{ccc}
1 & 0 & P(t) \\
0 & 1 & 0 \\
0 & 0 & 1
\end{array}\right), \quad P(t) \in \mathbf{Z}[t, 1 / t],
$$

as a central, infinitely generated subgroup. (This construction is due to Hall [1954, Theorem 7].)

Lemma 3.2. If $G$ is a $\sigma$-compact l.c. group, then it has a cocompact closed separable subgroup. 
Proof. By the Kakutani-Kodaira theorem, there is a compact normal subgroup $K$ such that $G / K$ is second countable. So $G / K$ admits a dense countable subset. Lift this subset to $G$, and let $D$ be the abstract (countable) group it generates. So $G=\overline{K D}=K \bar{D}$ since $K$ is compact. Thus $\bar{D}$ is cocompact; moreover, it is separable by construction.

Proof of Theorem 1.1. By Lemma 3.2, there is a cocompact closed separable subgroup in $A$. In other words, there is a homomorphism $f: Z \rightarrow A$ whose image has cocompact closure, where $Z=\mathbf{Z}^{(\omega)}$ is the restricted product of countably many copies of the infinite cyclic group. In view of Lemma 3.1, the group $Z$ can be embedded as a central subgroup of a finitely generated group $\Gamma$ (which can be chosen to be 3-solvable). The graph $F$ of $f$ is a closed discrete central subgroup of $\Gamma \times A$. Since $f$ is injective, it follows that the mapping of $A$ into $G=(\Gamma \times A) / F$ is injective. Moreover $A$ has open image (because the quotient map is open). So $A$ lies as an open (and central) subgroup of $G$. The latter group is compactly generated: indeed, the closure of the subgroup generated by a finite generating subset of $\Gamma$ is cocompact. By construction, if $A$ is second countable or totally disconnected, then so is $G$.

\section{Proof of Theorem 1.2}

Consider $B_{p}=\mathbf{Q}_{p} \rtimes_{p} \mathbf{Z}$, where the notation $\rtimes_{p}$ means that the $\mathbf{Z}$-action is through multiplication by powers of $p$. Let $A$ be the group of adeles; that is, the set of elements in $\prod_{p} \mathbf{Q}_{p}$ ( $p$ ranging over all primes) whose projection in $\prod \mathbf{Q}_{p} / \mathbf{Z}_{p}$ is finitely supported, endowed with the ring topology for which $\prod \mathbf{Z}_{p}$ is a compact open subring. In the product $\prod_{p} B_{p}=\left(\prod_{p} \mathbf{Q}_{p}\right) \rtimes \prod_{p} \mathbf{Z}$, consider the subgroup $Z=\bigoplus_{p} \mathbf{Z}$ and endow it with the discrete topology. Finally, define $M=A \rtimes Z \subset$ $\prod_{p} B_{p}$. The group $M$ is metabelian and admits a unique Hausdorff group topology for which $\prod_{p} \mathbf{Z}_{p}$ is a compact open subgroup. In particular, $M$ is locally compact.

Theorem 1.2 is a consequence of the following:

Proposition 4.1. There is no embedding of $M$ as a closed subgroup of any compactly generated l.c. group.

More precisely, given any continuous homomorphism $f: M \rightarrow G$ to a compactly generated l.c. group $G$, there exists $p_{0}$ such that $\overline{f\left(\mathbf{Q}_{p}\right)}$ is a compact connected group for all $p \geq p_{0}$.

We begin with two lemmas on homomorphisms from $\mathbf{Q}_{p}$ into locally compact groups:

Lemma 4.2. For every continuous homomorphism $f: \mathbf{Q}_{p} \rightarrow G$ of $\mathbf{Q}_{p}$ to a connected-by-compact l.c. group $G$, the closure of the image $\overline{f\left(\mathbf{Q}_{p}\right)}$ is compact and connected. 
Proof. Assume first that $G$ is a virtually connected Lie group. Since $\mathbf{Q}_{p}$ is divisible, it has no nontrivial finite quotient. Thus $\overline{f\left(\mathbf{Q}_{p}\right)}$ is a closed abelian subgroup of a connected Lie group, so is isomorphic to a product $\Gamma \times \mathbf{R}^{k} \times T$ for some finitely generated abelian group $\Gamma$ and torus $T$. Invoking again that $\mathbf{Q}_{p}$ has no nontrivial finite quotient, we find $\Gamma=\{0\}$. Since $\mathbf{R}^{k} \times T$ has no small subgroup, the kernel of $f$ must be open in $\mathbf{Q}_{p}$. In particular, $f\left(\mathbf{Q}_{p}\right)$ is a torsion group, from which we infer that $k=0$. Therefore $\overline{f\left(\mathbf{Q}_{p}\right)}$ is a torus; in particular, it is compact.

Coming back to the general case, we now let $W$ be the maximal compact normal subgroup of $G$, which exists by Proposition 2.1(i). Proposition 4.1 ensures that $G / W$ is a virtually connected Lie group. By the special case above, we deduce that, denoting $K=\overline{f\left(\mathbf{Q}_{p}\right)}$, the group $K W / W$ is compact. Hence $K$ is compact as well. Since $K / K^{\circ}$ is profinite and $\mathbf{Q}_{p}$ has no nontrivial finite quotient, $K=K^{\circ}$; that is, $K$ is connected.

Remark 4.3. It follows from Pontryagin duality that $\mathbf{Q}_{p}$ has a continuous homomorphism with dense image into the circle, and also has an injective continuous homomorphism with dense image into the Pontryagin dual $\widehat{\mathbf{Q}}$ of $\mathbf{Q}$, which is a connected compact group.

Lemma 4.4. Every nontrivial continuous homomorphism $f: \mathbf{Q}_{p} \rightarrow G$ of $\mathbf{Q}_{p}$ to a totally disconnected l.c. group $G$ is proper, and either has a compact open kernel or is an isomorphism to its (closed) image.

Proof. We can suppose that $f$ has dense image, so $G$ is abelian. Let $U$ be a compact open subgroup in $G$. Then $f^{-1}(U)$ is an open subgroup of $\mathbf{Q}_{p}$. If it is all of $\mathbf{Q}_{p}$, then $U=G$, and since $U$ is profinite and $\mathbf{Q}_{p}$ has no nontrivial finite quotient, it follows that $U=\{1\}$, contradicting that $\mathrm{f}$ is nontrivial. Otherwise, $f^{-1}(U)$ is a compact open subgroup, so $f$ is proper and, in particular, has closed image and is the quotient map by some compact subgroup, giving the two possibilities.

Lemma 4.5. Every continuous homomorphism $f: B_{p} \rightarrow G$ of the group $B_{p}=$ $\mathbf{Q}_{p} \rtimes_{p} \mathbf{Z}$ to a totally disconnected l.c. group $G$ satisfies one of two alternatives: either $f$ is a topological isomorphism to its closed image, or $f\left(\mathbf{Q}_{p}\right)$ is trivial.

Proof. If $f\left(\mathbf{Q}_{p}\right)$ is nontrivial, then $f$ is proper in restriction to $\mathbf{Q}_{p}$ by Lemma 4.4. Since the only compact subgroup of $\mathbf{Q}_{p}$ that is normal in $B_{p}$ is the trivial group, it follows from Lemma 4.4 that the restriction of $f$ to $\mathbf{Q}_{p}$ is an isomorphism to its closed image.

Let $\Omega$ be the normalizer of $f\left(\mathbf{Q}_{p}\right)$ in $G$; this is a closed subgroup and there is a unique continuous homomorphism $\rho: \Omega \rightarrow \mathbf{Z}$ such that conjugation by $g \in \Omega$ on $f\left(\mathbf{Q}_{p}\right)$ multiplies the Haar measure by $p^{\rho(g)}$. In restriction to $\mathbf{Z}$, we see that $\rho \circ f$ is the identity. It follows that $f\left(\mathbf{Q}_{p}\right)$ is open in $f\left(B_{p}\right)$ and that $f$ is proper. 
Proof of Proposition 4.1. Let $f: M \rightarrow G$ be an arbitrary continuous homomorphism to a compactly generated 1.c. group $G$. Note that $G / G^{\circ}$ is a compactly generated totally disconnected 1.c. group. Therefore by Proposition 2.1(ii) it has a continuous proper action on a connected graph of degree $d$, for some $d$. By Lemma 2.4, for every $p>d$, the restriction to $\mathbf{Q}_{p}$ of the $G$-action on this graph has an open kernel. Hence, by Lemma 4.5, for all $p>d$ the action of $\mathbf{Q}_{p}$ on this graph is trivial.

Let $W / G^{\circ}$ be the (compact) kernel of the $G$-action on the graph. Thus $W$ is connected-by-compact and contains $f\left(\mathbf{Q}_{p}\right)$ for all $p>d$. In view of Lemma 4.2, this implies that for all $p>d$, the group $\overline{f\left(\mathbf{Q}_{p}\right)}$ is compact and connected.

Remark 4.6. Proposition 4.1 shows that there is no injective continuous homomorphism from $M$ to any totally disconnected compactly generated 1.c. group. On the other hand, $M$ admits an injective continuous homomorphism (not proper!) to a compactly generated l.c. group, which can be obtained as follows: start from the dense embedding $\mathbf{Q} \subset \mathbf{Q}_{p}$; it induces a dense embedding $\mathbf{Q}_{p} \subset \widehat{\mathbf{Q}}$, where $\hat{\mathbf{Q}}$ is the Pontryagin dual of $\mathbf{Q}$ (this is a compact connected group). Multiplication by $p$ is an automorphism of $\mathbf{Q}$ and thus induces a topological automorphism of $\widehat{\mathbf{Q}}$, also given by multiplication by $p$. So we obtain a continuous injective homomorphism $M \rightarrow \prod_{p} \widehat{\mathbf{Q}} \rtimes Z$, where the $p$-th component of $Z$ acts on the $p$-th component of the compact group $\prod_{p} \hat{\mathbf{Q}}$ by multiplication by $p$. By Proposition 1.5, the latter group $\prod_{p} \widehat{\mathbf{Q}} \rtimes Z$ embeds as a closed subgroup of a compactly generated 1.c. group.

\section{Some groups of permutations}

\section{A. A nonembedding criterion.}

Proposition 5.1. Let $H$ be a topologically simple totally disconnected locally compact group. Assume that $H$ has a compact open subgroup $K$ such that for every $k$, the group $K$ possesses for some prime $p>k$ a closed subgroup topologically isomorphic to a nontrivial pro-p-group (for example, $K$ has some element of order $p$ ). Then $H$ admits no nontrivial continuous homomorphism into any compactly generated locally compact group.

Proof. We use the criteria from Proposition 2.2 applied to $H$, in which we can replace "nontrivial" by "faithful" since $H$ is topologically simple. Thus, we only have to show

(1) $H$ has no faithful continuous action on any connected graph of bounded degree;

(2) $H$ has no faithful continuous representation into $\mathrm{GL}_{n}(\mathbf{C})$ for any $n$.

Condition (2) is immediate as $H$ has small nontrivial subgroups whereas $\mathrm{GL}_{n}(\mathbf{C})$ has none.

Now consider a continuous action of $H$ on a connected graph of bounded degree, say $\leq d$. Fix a vertex $x_{0}$. Then the stabilizer $K_{x_{0}}$ of $x_{0}$ in $K$ is open, hence of 
finite index in $K$. Therefore, the hypothesis implies that $K_{x_{0}}$, and hence also the full stabilizer $H_{x_{0}}$, contains a nontrivial pro- $p$-subgroup $L$ for some prime $p>d$. But Lemma 2.4 implies that $L$ acts trivially on the graph, so (1) holds.

5B. Proof of Theorem 1.3. Here we prove the continuous case of Theorem 1.3. The case of abstract homomorphisms is postponed to Section 5C.

There exist various sources of topologically simple groups satisfying the criterion of Proposition 5.1 and hence the conclusions of Theorem 1.3. We shall content ourselves with describing one of them, following a construction of Akin, Glasner and Weiss [Akin et al. 2008, §4]; we point out that those examples were independently obtained as part of a more general construction by Willis [2007, §3].

The construction goes as follows: Fix an infinite index set $J$ (we can have $J=\mathbf{N}$ in mind). Fix a family $u=\left(u_{k}\right)_{k \in J}$ of integers greater than 2 . Define the graph $\mathscr{G}=\mathscr{G}(u)$ (nonoriented, without self-loops) as a disjoint union of complete graphs $\mathscr{G}_{k}$ on $u_{k}$ elements; we denote the vertex set by $\mathscr{G}(u)$ as well. Let us call the height function $h$ the function $\varphi \rightarrow J$ mapping any $v \in \mathscr{G}_{k}$ to $k$. Note that $h$ completely characterizes the graph structure.

Given a self-map $f: \mathscr{G} \rightarrow \mathscr{G}$, we call a vertex $v \in \mathscr{G}_{u}$ singular if $h(f(v)) \neq u$. We call the self-map $f$ almost regular if only finitely many vertices are singular. If $f$ is a permutation, we say that $f$ is an almost automorphism of the graph with height function $(\varphi, h)$ if both $f$ and $f^{-1}$ are almost regular. The group of almost automorphisms of $(\varphi, h)$ is denoted by $S$ (or $S(u)$ if we need specify it). Its subgroup of automorphisms of $(\varphi, h)$ (consisting of those $f$ that preserve the height and the graph structure) is denoted by $K$ (or $K(u)$ ).

Note that $K$ is naturally isomorphic to the product $\prod_{k \in J} \operatorname{Sym}\left(u_{k}\right)$, which makes it a compact group. The group $S$ is endowed with the unique left-invariant topology making $K$ a compact open subgroup; this topology is obviously locally compact and is a group topology, as checked in [Akin et al. 2008, §4]. It is the union of an increasing filtering family of compact subgroups $\left(K_{F}\right)$, where $F$ ranges over finite subsets of $J$ and $K_{F}$ is defined as those elements in $S$ all of whose singularities and pairs of singularities lie in $\bigcup_{i \in F} \mathscr{G}_{i}$; note that $K_{\varnothing}=K$ and that $K_{F}$ is topologically isomorphic to

$$
\operatorname{Sym}\left(\sum_{i \in F} u_{i}\right) \times \prod_{k \in J \backslash F} \operatorname{Sym}\left(u_{k}\right) .
$$

Define $K_{F}^{+}$as its closed subgroup

$$
\operatorname{Alt}\left(\sum_{i \in F} u_{i}\right) \times \prod_{k \in J \backslash F} \operatorname{Alt}\left(u_{k}\right) .
$$

The filtering family $\left(K_{F}^{+}\right)$is increasing; we define $S^{+}$as an abstract group as the 
union $\bigcup_{n \in F} K_{F}^{+}$. Endow it with the left-invariant topology making $K_{\varnothing}^{+}$a compact open subgroup. For the same reason as for $S$, this is a group topology.

Finally, we define $A<S$ and $A^{+}<S^{+}$as the subgroups consisting of the finitary permutations, i.e., the permutations with finite support. Clearly $A$ is the group of all finitary permutations on the vertices of $\mathscr{G}$, while $A^{+}$is the index-two subgroup of $A$ consisting of the alternating finitary permutations.

Remark 5.2. It is easily seen that $A^{+}$and $A$ are dense as subgroups of $S^{+}$and $S$, respectively. Moreover, $A^{+}$is also dense in $S$ : indeed, since $A$ is dense, it is enough to show that any transposition $\left(\begin{array}{ll}x & y\end{array}\right)$ in $S$ can be approximated by a sequence of elements of $A^{+}$. This is indeed the case, using a sequence of double transpositions $\left(\begin{array}{ll}x & y\end{array}\right)\left(\begin{array}{ll}x_{n} & y_{n}\end{array}\right)$ with $x_{n}, y_{n}$ distinct elements of the same height $k_{n}$ and $n \mapsto k_{n}$ injective.

This implies in particular that the embedding of $S^{+}$into $S$, which is continuous, is not closed: indeed, its image is a proper subgroup which is dense since it contains $A^{+}$.

Remark 5.3. In [Akin et al. 2008], it is shown that $S$ has a dense conjugacy class under the assumption that the mapping $k \mapsto u_{k}$ has finite fibers (which implies that $J$ is countable). The precise statement of [ibid., Theorem 4.4] actually shows that such a conjugacy class can then be found inside $S^{+}$, and also shows that $S^{+}$itself admits a dense conjugacy class.

Let us show the following related but independent result. For the moment, the family $\left(u_{k}\right)$ of integers greater than 2 is arbitrary:

Proposition 5.4. Every nontrivial normal subgroup of $S^{+}$or $S$ contains $A^{+}$, and is thus dense. In particular, $S^{+}$and $S$ are both topologically simple.

Note that $S^{+}$and $S$ are not abstractly simple, since $A^{+}$is a proper dense normal subgroup in both.

Proof. Let $s$ be a nontrivial element in $S^{+}$or $S$ and $t \in A^{+}$. Let $N$ be the normal subgroup generated by $s$; we show that $t \in N$. For some finite subset $F$ of $J$ such that $\sum_{j \in F} u_{j} \geq 5$, the element $s$ belongs to $K_{F}$ and $t$ has support in the finite set $X=\bigcup_{i \in F} \varphi_{i}$ (which has at least 5 elements). The commutator $s^{\prime}$ of $s$ and a suitable element of $\operatorname{Alt}(X)$ is a nontrivial element of $N \cap \operatorname{Alt}(X)$. By simplicity of $\operatorname{Alt}(X)$, it follows that $t \in N$.

We deduce the following corollary, which implies Theorem 1.3:

Corollary 5.5. If $\left(u_{k}\right)_{k \in J}$ is unbounded, the groups $S(u)^{+}$and $S(u)$ admit no nontrivial continuous homomorphism into any compactly generated l.c. group.

Proof. We have to check that the hypotheses of Proposition 5.1 are fulfilled. The topological simplicity is ensured by Proposition 5.4. The local condition also 
holds: since $\left(u_{k}\right)$ is unbounded, every neighborhood of the identity contains finite symmetric groups of all orders and thus contains elements of all possible finite orders.

5C. Abstract homomorphisms of $S$ and $S^{+}$. We start with the following converse to Corollary 5.5:

Proposition 5.6. If $J$ is countable and $\left(u_{k}\right)_{k \in J}$ is bounded, then $S(u)^{+}$and $S(u)$ are both embeddable as open subgroups in compactly generated l.c. groups, which can be taken as topologically finitely generated.

Proof. The $\mathscr{G}_{k}$ form a countable partition of $\varphi$ by subsets of bounded cardinality, so there exists a permutation $\sigma$ of $\mathscr{G}$ globally preserving this partition and having finitely many orbits on $\mathscr{G}$. Then $\sigma$ normalizes $S$ and $S^{+}$as well as $K$ and $K^{+}$. Therefore the semidirect products $S \rtimes\langle\sigma\rangle$ and $S^{+} \rtimes\langle\sigma\rangle$ are well defined. They are totally disconnected locally compact groups containing $S$ and $S^{+}$respectively as open subgroups. Moreover they act naturally by permutations of $\mathscr{G}$. The subgroup generated by $A^{+}$and $\sigma$ is finitely generated (when $\sigma$ is transitive, this group was introduced by B. H. Neumann [1937, p. 127]). Since $A^{+}$is dense in $S$ and $S^{+}$, it follows that $S \rtimes\langle\sigma\rangle$ and $S^{+} \rtimes\langle\sigma\rangle$ are topologically finitely generated, hence compactly generated.

Using two theorems of S. Thomas, it is possible to improve Corollary 5.5 in the case where the sequence $\left(u_{k}\right)$ tends to infinity.

Theorem 5.7. Assume that $k \mapsto u_{k}$ has finite fibers. Then $S(u)^{+}$admits no nontrivial abstract homomorphism into any compactly generated l.c. group.

Proof. Since $J$ is countable, we suppose for convenience $J=\mathbf{N}$. We invoke the criterion from Proposition 2.2, applied to the group $S^{+}=S(u)^{+}$endowed with the discrete topology. Thus, it is enough to show that

(1) $S^{+}$has no nontrivial action on any connected graph of bounded degree;

(2) $S^{+}$has no nontrivial representation into $\mathrm{GL}_{n}(\mathbf{C})$.

Both conditions can be checked with the help of the following result: Consider the subgroup $L_{k}=\prod_{j \geq k} \operatorname{Alt}\left(u_{j}\right)$ of $S^{+}$. Observe that $S^{+}$is generated by the alternating finitary group $A^{+}$and $L_{k}$ (because $A^{+}$is dense), so it follows from Proposition 5.4 that $S^{+}$is normally generated by $L_{k}$.

Next, we use a result of Thomas [1999, Theorem 1.10] that every (abstract) subgroup of at most countable index in $L_{k}$ is open. This immediately shows that every action of $S^{+}$on a graph of at most countable valency is continuous, so (1) follows from the proof of Corollary 5.5 (which, through the proof of Proposition 5.1, shows that $S^{+}$admits no nontrivial continuous action on any connected graph of bounded valency). 
Suppose $S^{+}$has a nontrivial linear representation $\rho$ into some $\mathrm{GL}_{d}(\mathbf{C})$ over a field. Let $m_{i}$ be the dimension of the smallest nontrivial representation of the alternating group $\operatorname{Alt}(i)$; then $m_{i}$ tends to infinity (it can be shown that $m_{i}=i-1$ for $i \geq 7$, but a nice argument based on commutation [Abért 2006] gives a completely elementary lower bound $\simeq \sqrt{i}$ ). Fix $k$ so that $m_{u_{j}}>d$ for all $j \geq k$. Since $L_{k}$ normally generates $S^{+}$, the representation $\rho$ is nontrivial in restriction to $L_{k}$. By another result of Thomas [1999, Theorem 2.1], any nontrivial subgroup of $\mathrm{GL}_{d}(\mathbf{C})$ admits a subgroup of at most countable index. Apply this to $\rho\left(L_{k}\right)$ and let $H$ be its inverse image in $L_{k}$. By the choice of $k$, the kernel of $\rho$ contains the direct sum $\bigoplus_{j \geq k} \operatorname{Alt}\left(u_{j}\right)$, which is dense. So $H$ is dense; on the other hand, the first-mentioned result of Thomas implies that $H$ is open. We thus reach a contradiction.

We have seen in Corollary 5.5 that unboundedness of the sequence $\left(u_{k}\right)$ was sufficient to guarantee the absence of nontrivial homomorphisms of $S(u)$ or $S(u)^{+}$ to a compactly generated locally compact group. In contrast to this, the next result shows that the hypothesis that $\left(u_{k}\right)$ tends to infinity in Theorem 5.7 cannot be weakened to the unboundedness of the sequence:

Proposition 5.8. The quotient of the group $S$ (resp. $S^{+}$) by its subgroup of finitary permutations can be identified with

$$
\prod_{j \in J} \operatorname{Sym}\left(u_{j}\right) / \bigoplus_{j \in J} \operatorname{Sym}\left(u_{j}\right) \quad\left(\text { resp. } \prod_{j \in J} \operatorname{Alt}\left(u_{j}\right) / \bigoplus_{j \in J} \operatorname{Alt}\left(u_{j}\right)\right) .
$$

In particular:

(1) S has an uncountable abstract abelianization and has proper subgroups of finite index (such subgroups are necessarily dense).

(2) $S^{+}$has proper subgroups of finite index if and only if $k \mapsto u_{k}$ has an infinite fiber. It has a nontrivial abelianization if and only if $u^{-1}(\{3,4\})$ is infinite; and in this case the abelianization is uncountable.

Proof. The first statement follows from the fact that $S=A K$, so $S / A=A K / A=$ $K /(A \cap K)$; the argument for $S^{+}$is similar.

Denoting by $C_{p}$ the cyclic group of order $p$, the signature map induces a canonical surjection

$$
\prod_{j \in J} \operatorname{Sym}\left(u_{j}\right) / \bigoplus_{j \in J} \operatorname{Sym}\left(u_{j}\right) \rightarrow C_{2}^{J} / C_{2}^{(J)},
$$

proving that $S$ has an uncountable abelianization, and, by taking a suitable quotient, admits subgroups of index 2, proving (1). (Observe that $S^{+}$has index 2 in the kernel of the surjection $S \rightarrow C_{2}^{J} / C_{2}^{(J)}$.) 
Recall that $u_{k} \geq 3$ for all $k$. Concerning $S^{+}$, if $u$ has finite fibers, then, by Theorem 5.7, $S^{+}$has no nontrivial linear representation, and therefore has no proper subgroup of finite index. Also observe that if $F=u^{-1}(\{3,4\})$ is finite, then $S^{+}$is generated by the perfect groups $A^{+}$and $\prod_{j \notin F} \operatorname{Alt}\left(u_{j}\right)$ and thus is perfect.

Conversely, assume $u$ has a finite fiber $u^{-1}(\{m\})$. We obtain a surjective homomorphism

$$
S^{+} \rightarrow \operatorname{Alt}(m)^{J} / \operatorname{Alt}(m)^{(J)} .
$$

Taking the limit with respect to a nonprincipal ultrafilter yields a nontrivial finite quotient. Also, if $m \in\{3,4\}$, then $S^{+}$admits either $\operatorname{Alt}(3)^{J} / \operatorname{Alt}(3)^{(J)}$ or $\operatorname{Alt}(4)^{J} / \operatorname{Alt}(4)^{(J)}$ as a quotient, and thus admits either $C_{2}^{J} / C_{2}^{(J)}$ or $C_{3}^{J} / C_{3}^{(J)}$ as an uncountable abelian quotient.

\section{Proof of Theorem 1.4}

Our first example is the following: let $\mathbf{Q}$ be the Pontryagin dual of the discrete additive group $\mathbf{Q}$. So $\widehat{\mathbf{Q}}$ is a connected torsion-free compact group, and by Pontryagin duality, its automorphism group can be identified with the group of automorphisms of the group $\mathbf{Q}$, namely, the multiplicative group $\mathbf{Q}^{\times}$. The first example is then

$$
H_{1}=\widehat{\mathbf{Q}} \rtimes \Lambda,
$$

where $\Lambda$ is an arbitrary infinitely generated subgroup of $\mathbf{Q}^{\times}$endowed with the discrete topology (recall that $\mathbf{Q}^{\times}$is isomorphic to the product of its subgroup of order 2 and a free abelian group of countable rank).

Our second example is very similar in construction. Fix a prime $p$. Recall that the group $\mathbf{Z}_{p}^{\times}$is uncountable (it is known to be isomorphic to the product of a finite abelian group with $\mathbf{Z}_{p}$ ). Let $\Lambda$ be a countable infinitely generated subgroup of $\mathbf{Z}_{p}^{\times}$, and endow $\Lambda$ with the discrete topology. Our second example is

$$
H_{2}=\mathbf{Z}_{p} \rtimes \Lambda \text {. }
$$

A third example is

$$
H_{3}=\mathbf{R} \rtimes \Lambda,
$$

where $\Lambda$ is a countable infinitely generated subgroup of $\mathbf{R}^{\times}$; this is a Lie group.

Proposition 6.1. If an l.c. group $G$ admits an isomorphic copy of $H_{i}(i=1,2,3)$ as an open subgroup, then it admits a discrete quotient that is an infinitely generated abelian group. In particular, $G$ is not compactly generated.

Proof. Since the identity component $H^{\circ}=\widehat{\mathbf{Q}}$ is open in $H$, it is open in $G$ and thus $H^{\circ}=G^{\circ}$ is open and normal in $G$. Thus the action by conjugation of $G$ on $G^{\circ}=\widehat{\mathbf{Q}}$ defines a continuous homomorphism $\phi: G \rightarrow \mathbf{Q}^{\times}$that is the identity on $\Lambda$ and trivial on $G^{\circ}$. So $\operatorname{Ker}(\phi)$ is open and the image of $\phi$ contains $\Lambda$ and is thus 
an infinitely generated abelian group. This concludes the proof of the proposition for $H_{1}$. The proof for $H_{3}$ is similar.

Let us now deal with $\mathrm{H}_{2}$; since $\mathbf{Z}_{p}$ is not connected, the previous argument does not work. The subgroup $\mathbf{Z}_{p}$ being compact and open in $G$, it is commensurated by $G$; its abstract topological commensurator is the group $\mathbf{Q}_{p}^{\times}$, so $G$ naturally admits a continuous homomorphism to $\mathbf{Q}_{p}^{\times}$whose kernel contains $\mathbf{Z}_{p}$. Let us check this directly: observe that if $g \in G$, then there exists $n$ such that $g\left(p^{n} \mathbf{Z}_{p}\right) g^{-1} \subset \mathbf{Z}_{p}$, and then there exists a unique $\lambda(g)$, not depending on $n$, such that the conjugation by $g$, in restriction to $p^{n} \mathbf{Z}_{p}$, coincides with the multiplication by $\lambda(g)$. An immediate verification shows that $\lambda$ is a homomorphism. In restriction to $\Lambda$, the map $\lambda$ is the identity, and $\operatorname{Ker}(\lambda)$ is open in $G$ since it contains $\mathbf{Z}_{p}$. Thus $G / \operatorname{Ker}(\lambda)$ is a discrete abelian group containing $\Lambda$ and therefore fails to be finitely generated.

In order to conclude the proof of Theorem 1.4, it remains to show that those examples admit embeddings as closed subgroups into some compactly generated 1.c. groups.

For $H_{2}$, such an embedding can be obtained as follows. First embed $\Lambda$ into a finitely generated group $\Gamma$ (this is possible by Lemma 3.1). Thus $\Lambda$ can be diagonally embedded as a discrete subgroup into $\mathbf{Z}_{p}^{\times} \times \Gamma$. This embedding extends to a continuous embedding of $\mathbf{Z}_{p} \rtimes \Lambda$ into $\left(\mathbf{Z}_{p} \rtimes \mathbf{Z}_{p}^{\times}\right) \times \Gamma$. This second embedding is continuous and injective; moreover, it is proper, since it is a discrete embedding in restriction to the cocompact subgroup $\Lambda$.

An obvious similar construction works for the third example $\mathbf{R} \rtimes \Lambda$. However, both embeddings rely on the fact that $\Lambda$ is contained in a compactly generated 1.c. group of automorphisms of the normal subgroup (either $\mathbf{Z}_{p}$ or $\mathbf{R}$ ). For $H_{1}$, we use the following topological version of a classical theorem of Krasner and Kaloujnine [1951]:

Recall that given two groups $K$ and $Q$, the unrestricted wreath product $K \bar{\imath} Q$ is the semidirect product $K^{Q} \rtimes Q$, where $Q$ acts on $K^{Q}$ by shifting on the left, namely, $q \cdot f(r)=f\left(q^{-1} r\right)$. Assume now that $K$ is a topological group and $Q$ is a discrete group. Then the product topology on $K^{Q} \times Q$ makes $K \bar{\imath} Q$ a topological group.

Theorem 6.2. For every l.c. group $H$ that is an extension of a compact normal subgroup $K$ by a discrete quotient $Q$, there is an embedding of $H$ as a closed subgroup of the unrestricted wreath product $K \bar{\imath} Q=K^{Q} \rtimes Q$.

Proof of Proposition 1.5. Let $\Gamma$ be a finitely generated group containing $Q$. By Theorem 6.2, there is a closed embedding $H \leqslant K \bar{\imath} Q$. By the definition of the unrestricted wreath product, the embedding $Q \leqslant \Gamma$ extends to a closed embedding $K \bar{\imath} Q \leqslant K \bar{\imath} \Gamma$. 
Proof of Theorem 6.2. We begin by a general construction, not relying on the group topologies. Let $\pi: H \rightarrow Q$ be a surjective group homomorphism with kernel $K$. We will define, in a canonical way, a set $X=X(\pi)$ with commuting actions of $K \bar{\imath} Q$ and $H$ such that the $(K \bar{\imath} Q)$-action is simply transitive and the $H$-action is free. Given a choice of $x \in X$, this yields a unique injective homomorphism $F_{x}: H \rightarrow K \bar{\imath} Q$ mapping $h \in H$ to the unique element $s=F_{x}(h) \in K \bar{\imath} Q$ such that $h x=s^{-1} x$. The latter homomorphism depends on the choice of $x$ but is canonically defined up to postcomposition by inner automorphisms of $K \bar{\imath} Q$.

The set $X$ is defined to be the set of functions $f: Q \rightarrow H$ such that $\pi \circ f$ is a left translation of $Q$ by some element $\theta(f)$. Note that $X \neq \varnothing$; indeed, it contains the set of set-theoretic sections $Q \rightarrow H$ of $\pi$, which are the elements $f$ in $X$ such that $\theta(f)=1$.

Let $K^{Q}$ act on $X$ as follows. If $u \in K^{Q}$, define

$$
u \cdot f(q)=f(q) u(q)^{-1} .
$$

If $f \in X$, then $u \cdot f \in X$ and $\theta(u \cdot f)=\theta(f)$, because

$$
\pi \circ(u \cdot f)(q)=\pi\left(f(q) u(q)^{-1}\right)=\pi(f(q))=\theta(f) q .
$$

This is clearly an action.

Further, let $Q$ act on $X$ as follows. If $r \in Q$, define

$$
r \cdot f(q)=f\left(r^{-1} q\right) .
$$

Note that $\pi(r \cdot f(q))=\pi\left(f\left(r^{-1} q\right)\right)=\theta(f) r^{-1} q$, so $r \cdot f \in X$ and $\theta(r \cdot f)=$ $\theta(f) r^{-1}$.

We next claim that these actions define an action of the semidirect product $K \bar{\imath} Q$ on $X$. To verify the claim, we need to show that for all $f \in X, u \in K^{Q}$ and $r \in Q$, we have

$$
v \cdot f=r \cdot\left(u \cdot\left(r^{-1} \cdot f\right)\right),
$$

where $v \in K^{Q}$ is defined as $v: q \mapsto u\left(r^{-1} q\right)$. In other words, we have $v=r u r^{-1}$ in the wreath product $K \bar{\imath} Q$. Given $q \in Q$, we have

$$
v \cdot f(q)=f(q) v(q)^{-1}=f(q) u\left(r^{-1} q\right)^{-1} .
$$

On the other hand, we have

$$
\begin{aligned}
r \cdot\left(u \cdot\left(r^{-1} \cdot f\right)\right)(q) & =\left(u \cdot\left(r^{-1} \cdot f\right)\right)\left(r^{-1} q\right) \\
& =\left(r^{-1} \cdot f\right)\left(r^{-1} q\right) u\left(r^{-1} q\right)^{-1} \\
& =f\left(r r^{-1} q\right) u\left(r^{-1} q\right)^{-1} \\
& =f(q) u\left(r^{-1} q\right)^{-1},
\end{aligned}
$$


so $v \cdot f(q)=r \cdot\left(u \cdot\left(r^{-1} \cdot f\right)\right)(q)$ for all $q \in Q$, as desired.

A straightforward verification shows that the action of $K \bar{\imath} Q$ on $X$ that has just been defined is simply transitive.

Finally, the $H$-action on $X$ is defined as follows: if $g \in H$ and $f$ is a function $Q \rightarrow H$, define

$$
g \cdot f(q)=g f(q)
$$

If $f \in X$ and $g \in H$ and $q \in Q$, we have

$$
(\pi \circ(g \cdot f))(q)=\pi((g \cdot f)(q))=\pi(g f(q))=\pi(g) \pi(f(q))=\pi(g) \theta(f) q,
$$

so $g \cdot f \in X$ and $\theta(g \cdot f)=\pi(g) \theta(f)$.

We immediately see that the action of $H$, which is free, commutes with both the action of $K^{Q}$ and the action of $Q$, and thus commutes with the action of $K \bar{\imath} Q$. So, we have for $x \in X$ an injective homomorphism $F_{x}: H \rightarrow K \bar{\imath} Q$ as defined above.

Assume now that $K$ is a topological group, while $Q$ is still assumed to be discrete, so $K \bar{\imath} Q$ is a topological group. Endow $H^{Q}$ with the product topology, and endow $X \subset H^{Q}$ with the topology induced by inclusion, namely, the pointwise convergence topology. It is straightforward that the actions of $K \bar{\imath} Q$ and $H$ on $X$ are continuous and that orbital maps $K \bar{\imath} Q \rightarrow X$ are homeomorphisms. It follows that the homomorphism $F_{X}$ is continuous.

Let us now assume that $K$ is compact, so $K \bar{\imath} Q$ and $X$ are both locally compact. (As soon as $Q$ is infinite, the converse holds; namely, $K \bar{\imath} Q$ is locally compact if and only if $K$ is compact.) We claim that the homomorphism $F_{X}$ is then proper. Checking this amounts to verifying that the $H$-action on $X$ is proper: Let $U_{1}, U_{2}$ be nonempty compact subsets of $X$ and let us check that $I=\left\{g \in H: g U_{1} \subset U_{2}\right\}$ has compact closure. By compactness, $\theta\left(U_{2}\right)$ is finite, and therefore we deduce that $\pi(I)$ is finite. Since $\pi$ is proper, it follows that $I$ has compact closure.

\section{Acknowledgment}

We thank Pierre de la Harpe for discussions and in particular for pointing out the reference [Pestov 1986].

\section{References}

[Abels 1974] H. Abels, "Specker-Kompaktifizierungen von lokal kompakten topologischen Gruppen”, Math. Z. 135:4 (1974), 325-361. MR 49 \#9114 Zbl 0275.22011

[Abért 2006] M. Abért, "Representing graphs by the non-commuting relation", Publ. Math. Debrecen 69:3 (2006), 261-269. MR 2008j:05162 Zbl 1121.05056

[Akin et al. 2008] E. Akin, E. Glasner, and B. Weiss, "Generically there is but one self homeomorphism of the Cantor set", Trans. Amer. Math. Soc. 360:7 (2008), 3613-3630. MR 2008m:22009 Zbl 1144.22007 
[Hall 1954] P. Hall, "Finiteness conditions for soluble groups", Proc. London Math. Soc. (3) 4 (1954), 419-436. MR 17,344c Zbl 0056.25603

[Higman et al. 1949] G. Higman, B. H. Neumann, and H. Neumann, "Embedding theorems for groups", J. London Math. Soc. 24 (1949), 247-254. MR 11,322d Zbl 0034.30101

[Krasner and Kaloujnine 1951] M. Krasner and L. Kaloujnine, "Produit complet des groupes de permutations et problème d'extension de groupes, III", Acta Sci. Math. Szeged 14 (1951), 69-82. MR 14,242d Zbl 0045.30301

[Monod 2001] N. Monod, Continuous bounded cohomology of locally compact groups, Lecture Notes in Mathematics 1758, Springer, Berlin, 2001. MR 2002h:46121 Zbl 0967.22006

[Montgomery and Zippin 1955] D. Montgomery and L. Zippin, Topological transformation groups, Interscience, New York, 1955. MR 17,383b Zbl 0068.01904

[Neumann 1937] B. H. Neumann, "Some remarks on infinite groups", J. London Math. Soc. 1:2 (1937), 120-127. Zbl 0016.29501

[Neumann and Neumann 1959] B. H. Neumann and H. Neumann, "Embedding theorems for groups", J. London Math. Soc. 34 (1959), 465-479. MR 29 \#1267 Zbl 0563.33002

[Pestov 1986] V. G. Pestov, "On compactly generated topological groups", Mat. Zametki 40:5 (1986), 671-676, 699. In Russian; translated in Math. Notes 40:5 (1986), 880-882. MR 88j:22002 Zbl 0617.22001

[Schupp 1976] P. E. Schupp, "Embeddings into simple groups", J. London Math. Soc. (2) 13:1 (1976), 90-94. MR 53 \#5758 Zbl 0363.20026

[Thomas 1999] S. Thomas, "Infinite products of finite simple groups, II", J. Group Theory 2:4 (1999), 401-434. MR 2000m:20043 Zbl 0938.20025

[Willis 2007] G. A. Willis, "Compact open subgroups in simple totally disconnected groups", $J$. Algebra 312:1 (2007), 405-417. MR 2008d:22005 Zbl 1119.22005

Received November 28, 2012. Revised May 18, 2013.

Pierre-Emmanuel CAPrace

Departement de Mathematiques

UNIVERSITE CATHOLIQUE DE LOUVAIN, IRMP

CHEMIN DU CYCLOTRON 2

1348 LOUVAIN-LA-NEUVE

BELGIUM

pe.caprace@uclouvain.be

YVES CORNULIER

LABORATOIRE DE MATHÉMATIQUES

UNIVERSITÉ PARIS-SUD 11

BÂTIMENT 425

91405 ORSAY

FRANCE

yves.cornulier@math.u-psud.fr 


\title{
PACIFIC JOURNAL OF MATHEMATICS
}

\author{
msp.org/pjm
}

Founded in 1951 by E. F. Beckenbach (1906-1982) and F. Wolf (1904-1989)

\section{EDITORS}

Don Blasius (Managing Editor)

Department of Mathematics

University of California

Los Angeles, CA 90095-1555

blasius@math.ucla.edu

\author{
Paul Balmer \\ Department of Mathematics \\ University of California \\ Los Angeles, CA 90095-1555 \\ balmer@math.ucla.edu \\ Robert Finn \\ Department of Mathematics \\ Stanford University \\ Stanford, CA 94305-2125 \\ finn@math.stanford.edu \\ Sorin Popa \\ Department of Mathematics \\ University of California \\ Los Angeles, CA 90095-1555 \\ popa@math.ucla.edu
}

\author{
Vyjayanthi Chari \\ Department of Mathematics \\ University of California \\ Riverside, CA 92521-0135 \\ chari@math.ucr.edu \\ Kefeng Liu \\ Department of Mathematics \\ University of California \\ Los Angeles, CA 90095-1555 \\ liu@math.ucla.edu \\ Jie Qing \\ Department of Mathematics \\ University of California \\ Santa Cruz, CA 95064 \\ qing@ cats.ucsc.edu
}

\section{PRODUCTION}

Silvio Levy, Scientific Editor, production@msp.org

\section{SUPPORTING INSTITUTIONS}

ACADEMIA SINICA, TAIPEI

CALIFORNIA INST. OF TECHNOLOGY

INST. DE MATEMÁTICA PURA E APLICADA

KEIO UNIVERSITY

MATH. SCIENCES RESEARCH INSTITUTE

NEW MEXICO STATE UNIV.

OREGON STATE UNIV.

\author{
STANFORD UNIVERSITY \\ UNIV. OF BRITISH COLUMBIA \\ UNIV. OF CALIFORNIA, BERKELEY \\ UNIV. OF CALIFORNIA, DAVIS \\ UNIV. OF CALIFORNIA, LOS ANGELES \\ UNIV. OF CALIFORNIA, RIVERSIDE \\ UNIV. OF CALIFORNIA, SAN DIEGO \\ UNIV. OF CALIF., SANTA BARBARA
}

\author{
Daryl Cooper \\ Department of Mathematics \\ University of California \\ Santa Barbara, CA 93106-3080 \\ cooper@math.ucsb.edu \\ Jiang-Hua Lu \\ Department of Mathematics \\ The University of Hong Kong \\ Pokfulam Rd., Hong Kong \\ jhlu@maths.hku.hk \\ Paul Yang \\ Department of Mathematics \\ Princeton University \\ Princeton NJ 08544-1000 \\ yang@math.princeton.edu
}

These supporting institutions contribute to the cost of publication of this Journal, but they are not owners or publishers and have no responsibility for its contents or policies.

See inside back cover or msp.org/pjm for submission instructions.

The subscription price for 2014 is US $\$ 410 /$ year for the electronic version, and \$535/year for print and electronic.

Subscriptions, requests for back issues and changes of subscribers address should be sent to Pacific Journal of Mathematics, P.O. Box 4163, Berkeley, CA 94704-0163, U.S.A. The Pacific Journal of Mathematics is indexed by Mathematical Reviews, Zentralblatt MATH, PASCAL CNRS Index, Referativnyi Zhurnal, Current Mathematical Publications and Web of Knowledge (Science Citation Index).

The Pacific Journal of Mathematics (ISSN 0030-8730) at the University of California, c/o Department of Mathematics, 798 Evans Hall \#3840, Berkeley, CA 94720-3840, is published twelve times a year. Periodical rate postage paid at Berkeley, CA 94704, and additional mailing offices. POSTMASTER: send address changes to Pacific Journal of Mathematics, P.O. Box 4163, Berkeley, CA 94704-0163.

PJM peer review and production are managed by EditFLOW ${ }^{\circledR}$ from Mathematical Sciences Publishers.

\section{PUBLISHED BY}

\section{mathematical sciences publishers \\ nonprofit scientific publishing}

http://msp.org/

(C) 2014 Mathematical Sciences Publishers 


\section{PACIFIC JOURNAL OF MATHEMATICS}

Volume $269 \quad$ No. $2 \quad$ June 2014

Totaro's question for simply connected groups of low rank

257

JODI BLACK and RAMAN PARIMALA

Uniform hyperbolicity of the curve graphs

269

BRIAN H. BOWDITCH

Constant Gaussian curvature surfaces in the 3-sphere via loop groups

281

DAVID BRANDER, JUN-ICHI INOGUCHI and SHIMPEI KOBAYASHI

On embeddings into compactly generated groups

305

PIERRE-EMmanuel CAPRACE and Yves CORNULIER

Variational representations for $N$-cyclically monotone vector fields

ALFRED GALICHON and NASSIF GHOUSSOUB

Restricted successive minima

341

MaRTIN HenK and CARSTEN THIEL

Radial solutions of non-Archimedean pseudodifferential equations

355

ANATOLY N. KochubeI

A Jantzen sum formula for restricted Verma modules over affine Kac-Moody

algebras at the critical level

JOHANNES KÜBEL

Notes on the extension of the mean curvature flow

YAN LENG, ENTAO ZHAO and HaORAN ZHAO

Hypersurfaces with prescribed angle function

Henrique F. DE Lima, ERAldo A. Lima JR. and Ulisses L. Parente

Existence of nonparametric solutions for a capillary problem in warped products

JoRGE H. LiRA and GABRIELA A. WANDERLEY

A counterexample to the simple loop conjecture for $\operatorname{PSL}(2, \mathbb{R})$

425

KATHRYN MANN

Twisted Alexander polynomials of 2-bridge knots for parabolic representations

TAKAYUKI MORIFUJI and ANH T. TRAN

Schwarzian differential equations associated to Shimura curves of genus zero

FANG-TING TU

Polynomial invariants of Weyl groups for Kac-Moody groups 